\title{
Moda Virtual: aceleração no processo de transfor- mação digital devido à pandemia de COVID-19
}

\author{
Wadson Gomes Amorim;
}

Rachel Sager Boldt

\section{resumo:}

A pandemia global de 2020 levou o sistema da moda à uma nova crise. Embora a sua duração e a gravidade permaneçam desconhecidas é evidente que essa indústria está apenas no começo de sua luta. Neste artigo, temos o objetivo de apresentar e discutir o papel das novas soluções tecnológicas ligadas ao conceito de Indústria 4.0 frente à crise gerada pela pandemia de Covid-19. A redação do trabalho foi possível a partir de uma revisão sistemática da literatura relacionada às tecnologias digitais emergentes na indústria da moda e a análise de notícias recentes que apontaram possíveis aplicações como soluções alternativas para as incertezas do agora. $O$ estudo, realizado entre maio e agosto de 2020, considera contextos da indústria, pesquisa e ensino. Explora uma série de exemplos de tecnologias em uso nesses contextos, revelando novos fluxos de trabalho e oportunidades resultantes do uso de softwares 3D no processo de Design. Argumenta que as vantagens das tecnologias digitais para melhorar o processo de Design de Moda são particularmente evidentes diante desse momento adverso que vivemos.

\section{palavras-chave:}

Indústria da Moda; distanciamento social; virtualização; Softwares 3D 


\section{Introdução}

Assim que a pandemia global do novo coronavírus se instaurou, o impacto sobre as atividades da indústria da moda foi imediato. Devido às preocupações com a saúde pública e as medidas de distanciamento social, dentro de pouco tempo, governos em todo o mundo exigiram o fechamento de fábricas, lojas e o cancelamento de eventos, com o intuito de retardar a propagação do vírus. Isso tem interferido tanto no macro quanto nos micros níveis da vida social, da alta costura ao vestuário de massa, da produção de matérias-primas às redes de distribuição. A crise gerada pela pandemia tem consequências que são, simultaneamente, econômicas, técnicas, culturais, estéticas e políticas, em contextos locais, nacionais e globais. Diante deste cenário, a indústria da moda no Brasil e do mundo enfrentam desafios objetivos nos sistemas de produção, distribuição e venda (ABIT, 2020; AMED et al., 2020).

Nos últimos anos testemunhamos mudanças sem precedentes na indústria da moda, guiadas principalmente pelo processo de transformação digital (BRUNO, 2017). Entretanto, este processo está sendo inevitavelmente acelerado pelo contexto excepcionalmente imposto pela pandemia. Como as medidas de controle sanitário impediram o contato físico tanto nas etapas internas quanto externas da cadeia de suprimentos - e provavelmente ainda colocará limitações nos processos manuais, nos encontros físicos e nas viagens por algum tempo - a indústria da moda passou a explorar opções de migração para o espaço virtual em toda a sua cadeia de valor. Essa migração contempla propostas de virtualização de experiências intermediadas por mídias não tradicionais. A inovação através de tecnologias digitais não é novidade para essa indústria, no entanto, o impacto devastador da crise as colocou em foco, dada a necessidade imediata de soluções alternativas. Como designers, observamos as limitações e as ferramentas emergentes com grande interesse para propor novas soluções.

Com o desenvolvimento da ciência e da tecnologia no século XXI houve uma expansão das tecnologias de informação, comunicação e produção. No campo do Design de Moda, uma variedade de pesquisas e ensaios ligados à transformação digital estão em andamento neste momento. Quando falamos em digitalização dos processos na moda, estamos nos referindo principalmente à substituição das práticas físicas e manuais tradicionais por tecnologias que promovam maior eficiência e sustentabilidade ambiental, processadas em ambiente virtual e de fácil comunicação/colaboração via internet. Hoje, no desenvolvimento do vestuário, já é possível explorar tecnologias que incluem o desenho e a manufatura assistidos por computadores; a modelagem plana com gradação automática paramétrica a; estudos antropométricos via big data (COSTA; PIERRE; LUISA, 2015; LIU et al., 2018); o diagnósticos de tendência de estilo baseados em inteligência artificial (MA et al., 2020); a criação imagética auxiliada por algoritmos de base GAN (Generative Adversarial Network) e CAN (Creative Adversarial Networks) (ELGAMMAL et al., 2017; KHERDE1; JAWADE2, 2018); o planejamento de coleção intermediada por inteligência artificial (WONG; CHAN, 2001); a simulação digital das propriedades físicas de tecidos e avaliação do fit em corpos reais digitalizados em scanners 3D; a produção digital doméstica e a impressão 3D (BOLDT et al., 2019; BRUNO, 2017; KANG; LEE; KIM, 2020; KIM et al., 2020; LU; MOK; JIN, 2017; SADAT; SAYEM, 2015). Esses e outros novos processos tecnológicos integram o conceito relacionado à "Quarta Revolução Industrial", também denominada como "Indústria 4.0".

O termo "Indústria 4.0" começou a ser difundido em 2011, quando o governo alemão o anunciou como uma de suas principais iniciativas estratégicas em alta tecnologia. Desde então, há um crescente interesse pelo tópico, tanto por pesquisadores, quanto empresários motivados pelos possíveis impactos disruptivos nas esferas econômicas, sociais, culturais e técnicas causadas pelas promessas deste modelo (BRUNO, 2017; DRATH; HORCH, 2014; HERMANN; PENTEK; OTTO, 2016). Klaus Schwab, engenheiro e economista alemão criador do Fórum Econômico Mundial, formalizou os conceitos que caracterizam a quarta revolução industrial. Schwab (2016) diferencia a Indústria 4.0 das demais revoluções tecnológicas a partir de 3 pilares: (1) velocidade na evolução tecnológica em ritmo exponencial; (2) amplitude e profundidade, sustentadas pelas tecnologias digitais provocando mudanças paradigmáticas na forma, produção e papel individual no contexto industrial; e (3) impacto sistêmico, nos âmbitos industriais, políticos e sociais. 
Neste artigo temos como objetivo apresentar e discutir o papel das novas soluções tecnológicas ligadas ao conceito de Indústria 4.0 frente à crise gerada pela pandemia de Covid-19 no ano de 2020 . A redação deste trabalho foi possível a partir de uma revisão sistemática da literatura relacionada às tecnologias digitais de uso emergente na indústria da moda, e a análise de notícias recentes relacionadas às suas possíveis aplicações como soluções alternativas diante da crise instaurada. $\mathrm{O}$ estudo realizado entre maio e agosto de 2020, considera contextos da indústria, pesquisa e ensino. O artigo explora uma série de exemplos de tecnologias em uso nesses contextos e revela novos fluxos de trabalho e oportunidades resultantes do uso de softwares 3D no processo de design. Argumenta que as vantagens das tecnologias digitais para melhorar o processo de Design de Moda são particularmente evidentes diante desse momento adverso em que vivemos.

\section{A Moda na Pandemia}

A primeira temporada internacional de moda completamente alterada pela pandemia do Covid-19 aconteceu em julho de 2020. Nela, as empresas do setor utilizaram diferentes recursos digitais para apresentar suas coleções, como a transmissão ao vivo e on-line de desfiles e lookbooks físicos ou vídeos de produtos transportados para o ambiente 3D (FRIEDMAN, 2020). Alguns dos momentos mais comentados pela mídia especializada foram protagonizados por marcas menores e mais jovens, como a italiana Sunnei, a londrina Ralph \& Russo, a japonesa Undercover e a congolesa Hanifa, que alcançaram ampla publicidade das suas apresentações.

A Sunnei, uma marca de moda de Milão, lançou a coleção Primavera/Verão SS21 de forma digital, trazendo surpresas inesperadas ao público. Eles investiram na tecnologia 3D com modelos virtuais, dividindo a coleção em duas partes. A primeira apresentou 20 looks brancos usados por 5 avatares diferentes dançando Macarena em um desfile virtual. A segunda lançou um novo projeto chamado Sunnei Canvas, que tem como foco a personalização da peça pelo cliente. Por meio de uma plataforma exclusiva é permitido ao comprador a escolha do tecido, da cor e da estampa, conferindo 1.200 combinações possíveis das peças dentro das opções disponíveis. Espera-se que este modelo de negócios inovador traga uma experiência diferente, o que pode reduzir o desperdício excessivo causado por roupas que não são produzidas sob demanda. A marca trabalhou em parceria com os engenheiros 3D da Pezzo di Studio para criar um novo mundo de possibilidades para a empresa e seus clientes. Esse processo complicado, que normalmente levaria muitos meses para ser executado, entretanto, foi produzido em apenas três meses (BLANKS, 2020). A figura 01 exibe um dos frames do vídeo de apresentação da coleção digital Sunnei SS21.

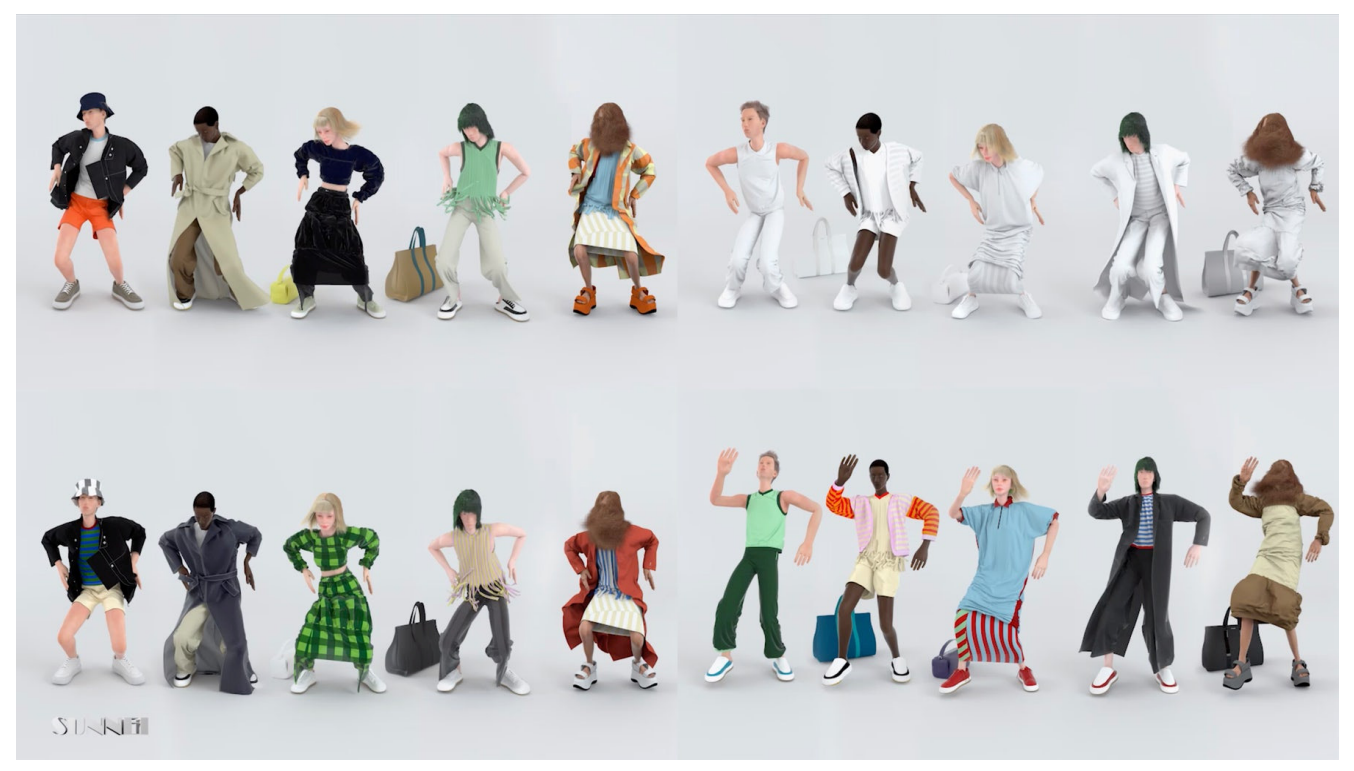

Figura 01. Coleção digital Sunnei 2020

Fonte: Sunnei, 2020 
A Ralph \& Russo, em parceria com a Federação Francesa de Alta Costura, apresentou sua coleção de Outono/Inverno 2020/2021 na forma de uma experiência digital que incentivou uma viajem virtual pelo mundo (SEGATTI, 2020). Combinando a tecnologia com elementos criativos, as peças inicialmente projetadas nos ateliês parisienses foram modeladas em 3D e ambientadas em paisagens turísticas de diversas partes do globo. A Undercover também criou avatares para mostrar 42 looks que podem ser vistos de qualquer ângulo em seu site (FFW, 2020). A marca Hanifa faria sua apresentação no New York Fashion Show, mas devido ao cancelamento do evento, apresentou sua coleção digitalmente via Instagram (CAMPBELL, 2020). Curiosamente, esse desfile não mostra nenhuma característica física de um modelo humano, mas mostra as roupas sobre um corpo moldado que parece ser feito de ar. Os modelos podem ser vistos andando nos posts no Instagram, onde exibem referências africanas como cores vivas, dobras e tecidos esvoaçantes, além de fazer uma crítica às atividades mineradoras ilegais que ocorrem na República do Congo (VIEIRA, 2020). A imagem a seguir (figura 02) mostra exemplos das coleções digitais supracitadas (Ralph \& Russo, 2020; Undercover ,2020; Hanifa, 2020).
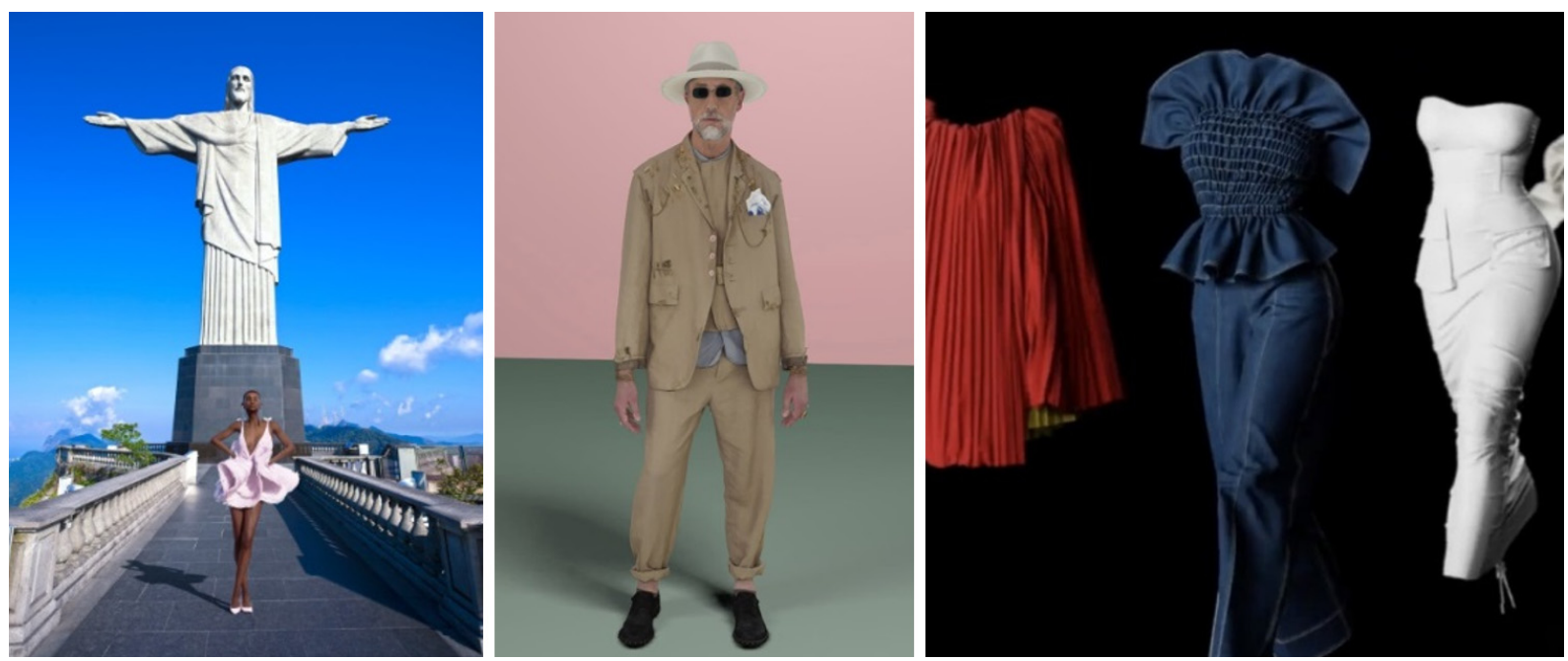

Figura 02. Respectivamente Ralph \& Russo, Undercover, Hanifa

Fonte: Ralph \& Russo, 2020; Undercover ,2020; Hanifa, 2020

As iniciativas foram bem recebidas pelo público, pois além de apresentarem o conceito das coleções, as ações evidenciaram o respeito pelas recomendações de distanciamento social, respeitando à saúde dos envolvidos na construção do show. A apresentação da marca Hanifa teve uma grande repercussão nas redes sociais, além de ser a primeira no calendário, apresentou sua coleção de forma inesperada. As roupas, mesmo que virtuais, foram protagonistas no show e a figura oculta das modelos evidenciou ainda mais as peças, os acabamentos e os movimentos dos tecidos.

\section{Novas Relações com o Consumidor}

Nos encontramos no início de uma nova fase para os produtos de moda. Com a virtualização dos produtos, a experiência em moda foi ampliada, os canais e formas de contato estão em expansão e em experimentação. A virtualização pode aprimorar a experiência existente, permitindo que as peças de vestuário sejam vistas de qualquer ângulo, com a maior quantidade de detalhes, além de exibir as habituais informações sobre o tamanho e o preço. A interação pode se dar em diferentes plataformas móveis, web ou mesmo em espaços virtuais, que podem promover experiências imersivas e interativas.

Serviços especializados buscam diferentes formas de colocar estilistas/fabricantes em contato com o público consumidor. Como os exemplos dos showrooms digitais das empresas Hatch e VOOR, que hospedam amostras digitais criadas com o software de design 3D; sem nenhum produto físico envolvido. O toque e a sensação do tecido são aspectos importantes para uma peça de roupa, o que a 
tecnologia digital ainda não é capaz de fornecer. Esse desafio terá que ser enfrentado para que os showrooms digitais sejam totalmente adotados. Desenvolvimentos recentes de luvas hápticas e telas especiais sensíveis ao toque podem "digitalizar" a textura das amostras de tecido, permitindo que os usuários "sintam" o produto no futuro (LEY, 2020).

O serviço de realidade virtual da Ordre se propõe a colocar os compradores na primeira fila, a partir do conforto de suas casas, com óculos e fones imersivos de VR (virtual reality) construindo experiências alternativas (DOUPNIK, 2018). O software de inteligência artificial da Bigthinx realiza exames de corpo em 3D com apenas um smartphone. Ao utilizar fotos comuns dos corpos dos clientes, eles podem criar avatares 3D personalizados e roupas digitais para ensaios virtuais e desfiles de moda. Além disso, quando um consumidor cria seu próprio avatar, ele também pode ver como essa peça se encaixaria em uma simulação de seu próprio corpo, permitindo que as redes neurais da Bigthinx possam usar essas imagens para ajudá-lo a encontrar o tamanho ideal (KHOSLA, 2020). Produtos da startup HoloMe permitem que os usuários visualizem imagens pré-gravadas ou transmissões ao vivo em seus dispositivos inteligentes, com imagens holográficas sobrepostas em seu próprio ambiente (LEY, 2020).

\section{O 3D na Moda Pré-pandemia}

Apesar de sua disponibilidade comercial há mais de 15 anos, podemos considerar que a simulação têxtil e prototipagem virtual de vestuário, elaborada por meio de sistemas $\mathrm{CAD} 3 \mathrm{D}$, possui ainda uma utilização reduzida no contexto do design de produtos de base têxtil. Não sendo considerada ainda um artifício intrínseco ao processo de desenvolvimento de vestuário, como ocorre em outras categorias de produtos. Entendemos que, as principais aplicações comerciais dos sistemas, são destinadas ao suporte criativo, apoio ao desenvolvimento técnico para validação de produtos, marketing e vendas (SADAT; SAYEM, 2015). Em um recorte pré-pandêmico, destacamos algumas iniciativas e depoimentos de empresas que utilizaram o recurso virtual no desenvolvimento e promoção dos seus produtos.

Em 2015 foi apresentada a campanha Primavera/Verão 2016 da Louis Vuitton campanha batizada de "Série 4". Ela exibe personagens do videogame Final Fantasy usando simulações em 3D das peças da marca (LOUIS VUITTON, 2015). Outro exemplo é a ação colaborativa entre a Balmains e a Shudo, no desenvolvimento da primeira modelo $100 \%$ digital. Nesta ação, foi desenvolvida a campanha Virtual Army onde os designers digitais recriaram o vestuário e as bolsas através da simulação 3D, usando os padrões de modelação reais da coleção Pré-outono de 2018 da Balmains (CLO VIRTUAL FASHION, 2018). Imagens das coleções "Serie 4" (figura 03) e "Virtual Army" (figura 034), podem ser vistas logo a seguir.

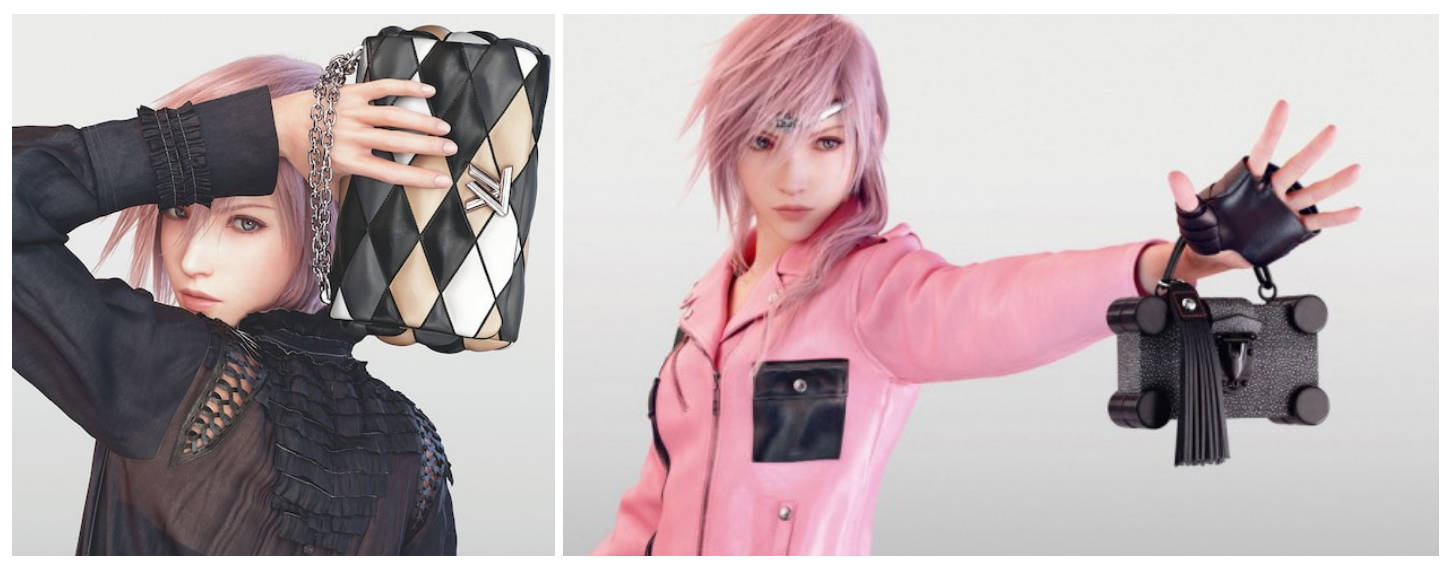

Figura 03. Coleção Serie 4 Primavera-Verão 2016 da Louis Vuitton

Fonte: Louis Vuitton, 2015 


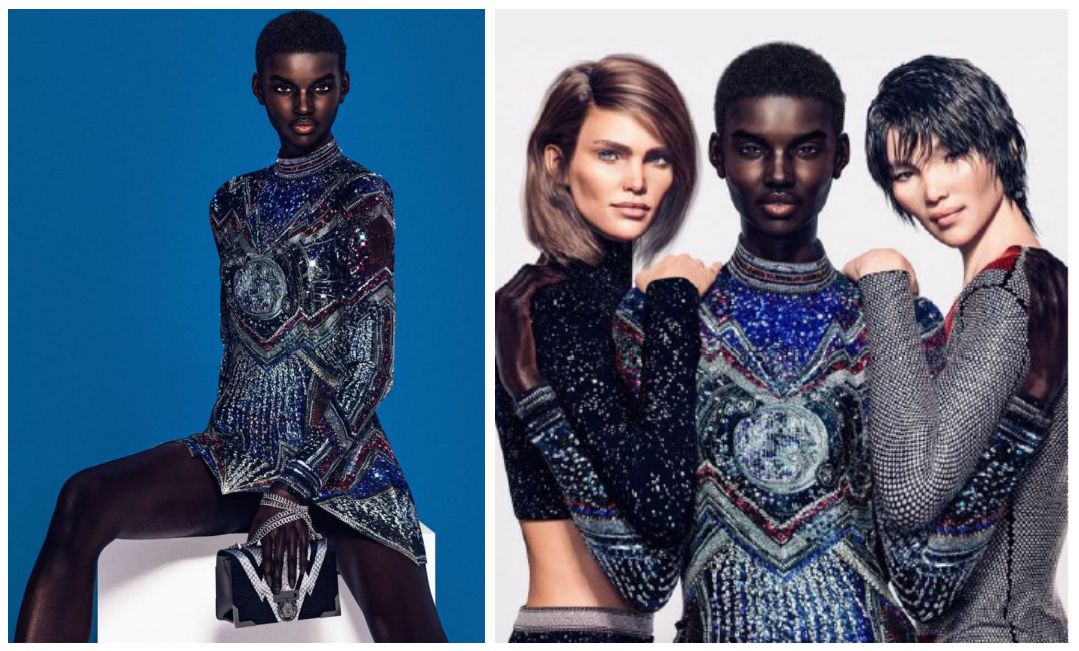

Figura 04. Coleção digital Virtual Army de Balmains

Fonte: Balmains, 2018; Clo Virtual Fashion, 2018

A marca Tommy Hilfiger, em abril de 2020, já contava com $66 \%$ dos seus designers capacitados em tecnologias os permitiam produzircerca de $80 \%$ dos projetos em 3D. A empresa almeja digitalizar toda sua cadeia de valor, desde os aspectos relacionados à promoção dos conceitos visuais, até os contributos técnicos e ergonômicos passíveis de serem adicionadas ao vestuário. Segundo Hilfiger, a virtualização contribui com a união dos colaboradores e promove novos patamares de criatividade (SOCHA, 2020).

A empresa portuguesa Cordeiro Campos, que atua no desenvolvimento e confecção de produtos para o conglomerado de empresas têxteis espanhol Inditex, e outras redes varejistas europeias, diz que: "A tecnologia 3D, ajudou-nos a remover ineficiências e a reduzir o nosso tempo de lançamento no mercado". São também ressaltadas as questões relativas à possibilidade de verificar o fit e o caimento do tecido, durante o processo de concepção do projeto, como uma mais valia da utilização da tecnologia (LECTRA, 2018). Já a empresa Alberto Lovisetto, onde o designer Dainese, especialista em vestuários técnicos para motociclistas, relata que com o uso do sistema $3 \mathrm{D}$, os projetos são aprovados em $90 \%$ dos casos, com a execução de apenas uma peça piloto. Para Silvio Cattarin, diretor da repartição CAD da empresa Italiana Emilio Pucci, o uso do recurso 3D possibilitou a redução de $30 \%$ de tempo consumido no processo de prototipagem. Para YuJia Fang, designer da empresa de moda Kashion, a tecnologia 3D oferece um meio eficiente para a discussão entre o designer e o modelista (CLO VIRTUAL FASHION, [s.d.]). A marca Roberto Cavalli, ressalta as facilidades trazidas pelo uso do sistema como a redução de custo e tempo na execução do desenvolvimento e produção com a manutenção dos padrões de qualidade. Representantes da Scott Sports relatam que o sistema o auxilia na percepção de ideias abstratas do projeto nas fases iniciais de desenvolvimento, e da Under Armour complementam que é dada a possibilidade de comunicação transversal entre as diferentes capacidades das equipes envolvidas (OPTITEX, [s.d.]).

\section{O Projeto Virtual Além da Imagem}

$\mathrm{Na}$ indústria da moda, geralmente, o projeto do vestuário é executado por uma equipe de profissionais que dominam determinadas etapas do processo de criação, produção e distribuição. Esse projeto não se limita apenas a esboçar desenhos para comunicar desejos de moda, mas também se concentra em processos técnicos, como a elaboração de modelagens, seleção de materiais e processos produtivos das roupas.

A modelagem geralmente é uma responsabilidade do modelista, cuja tarefa é planejar a materialização da roupa, o que tradicionalmente acontece por meio da elaboração de moldes bidimensionais em papel (modelagem plana) ou através de estudos tridimensionais com tecidos sobre manequim (Draping ou Moulage). Contudo, com a complexidade e o grande volume de arquivos 
provenientes do processo tradicional de desenvolvimento de vestuário, se fez necessária a criação de novos meios para projetar e organizar modelagem em ambiente digital (ALDRICH, 2008). O design de roupas assistidas por computadores vem se desenvolvendo desde o final dos anos 1980 (MENG; MOK; JIN, 2012), e tem acelerando o processo de confecção na indústria da moda desde então (MAO et al., 2011; PAGE, 2013). Com o surgimento de sistemas CAD (Computer Aided Design) e CAM (Computer Aided Manufacturing) utilizáveis no campo da moda, a informatização passou a atuar principalmente na substituição dos processos manuais nos setores de criação, modelação e corte (SILVEIRA; SILVA, 2011).

O principal objetivo ao criar modelagens usando softwares é aumentar a eficiência e a precisão, sem o uso de materiais físicos convencionais, já que os arquivos são armazenados em formato digital. Atualmente a contribuição dos sistemas CAD está sendo ampliada por novas possibilidades de interação. Isso tem permitido o desenvolvimento de padrões de modelação $100 \%$ digitais, parametrização das modelações em função de medida individuais (COSTA; PIERRE; LUISA, 2015; HONG et al., 2018), criação de bases de modelagem por meio da extração da superfície de modelos 3D digitalizados (BOLDT et al., 2018), avaliação do fit através de provas digitas (LIU et al., 2017), além da simulação e visualização de imagens do produto e minimização de perdas antes de produção (MCQUILLAN, 2020).

Durante o projeto de uma peça de roupa pode haver várias alterações. Antes, protótipos e peças pilotos precisavam ser refeitos a cada alteração para avaliação e validação dos modelos, resultando na produção de várias amostras e em prazos mais longos. Em um passado recente, os designers técnicos usavam os sistemas CAD para fazer melhorias digitais iterativas em modelagens $2 \mathrm{D}$ e ajustá-las antes de conceber uma amostra física. Hoje, o vínculo entre simulação 3D e modelagem 2D pode permitir que eles cortem, ajustem e simulem o produto virtualmente e à distância, com precisão, e sem a necessidade de utilizar materiais físicos durante o processo criativo. Muitas soluções de softwares de design 3D oferecem feedback em tempo real, incluindo informações sobre o preço de custo do produto, calculadas a partir do uso de matérias primas, e estimativa de tempo de produção. Isso permite que alterações sejam feitas imediatamente, testando e repetindo operações sem a necessidade da confecção de amostras físicas. A natureza colaborativa da criação de roupas digitais em plataformas 3D provou que também é possível melhorar a comunicação entre equipes, aumentando os ciclos de feedback e a eficiência em toda a cadeia de valor (MCQUILLAN, 2020).

A beleza do 3D, para além da aparência e desempenho técnico de produto, é que, uma vez que exista uma peça com a modelagem precisa, ela está pronta para ser usada em diversos contextos. A peça 3D pode substituir a modelagem em papel ou tecido durante a criação, ser visualizada e manipulada por designers técnicos em tempo real para melhorar o ajuste e desenvolver variações formais. Além disso, posteriormente, essa modelagem pode ser vestida e renderizada em um avatar foto realista, com qualidades que podem ser usadas na publicidade do produto, ou até mesmo substituir um produto físico em um catálogo de comércio eletrônico, como no exemplo apresentado na figura 05 .

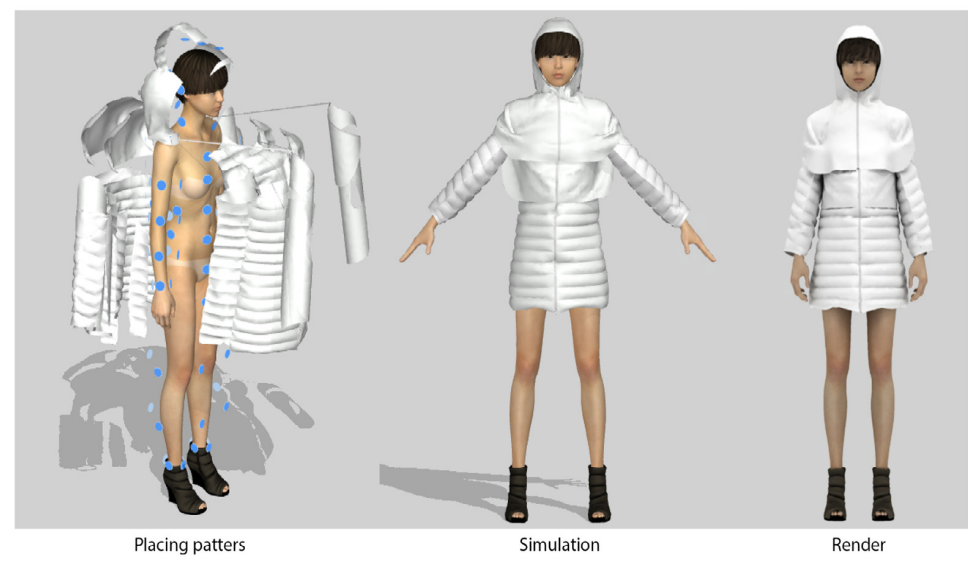

Figura 05: Processo de criação 3D de vestuário por Clo3D 
Há hoje no mercado vários softwares dedicados à simulação do vestuário em $3 \mathrm{D}$, como o Vstitcher ${ }^{\mathrm{TM}}$, desenvolvido pelos israelitas da Browzwear, o 3D Runway, desenvolvido pela OptiTex International, o Accumark 3D, desenvolvido pelos norte-americanos da Gerber, o TUKA3D, desenvolvido pela Tukatech, Modaris 3D, desenvolvido pelos franceses da Lectra, o Vidya, desenvolvido pelos alemães da Assyst, o Clo 3D e o Marvelous Designer, desenvolvido pela coreana Clo Virtual Fashion, e o brasileiro Audaces 4D.

Novos negócios de moda emergem dessa nova realidade. A terceirização surge como alternativa à capacitação dos profissionais internos. Os processos decorrentes da modelagem 3D podem ser executados por empresas especializadas em design digital, como Pixelpool; que declara poder criar até 500 peças de vestuário digitais por dia a Virtuality.Fashion que se propõe a construir uma coleção digital inteira em menos de uma semana; a Change of Paradigm oferece diferentes tipos de experiências de realidade aumentada, comércio digital padrão, experiência na loja e loja virtual por meio do 3D.

Os softwares 3D, em sua maioria, possuem bibliotecas virtuais de amostras de tecidos, estes simulam a estética e os aspectos relacionados à física dos artigos têxteis. Deste modo, abriu-se um mercado para a digitalização e simulação de amostras têxteis, dada a renovação e inovação constante do setor, a fim de viabilizar a simulação das coleções de forma realista nos âmbitos estético e funcional. Plataformas especializadas como a Vizoo e a Seddi oferecem este serviço de digitalização. Com uma coleção cada vez maior de materiais, texturas e amostras, a empresa Swatchbook fornece o armazenamento em nuvem que permite que marcas e fabricantes criem uma biblioteca centralizada que possa ser facilmente visualizada, explorada e compartilhada.

Com base nas modelagens digitais criadas na fase de design, as empresas de moda são capazes de compilar a coleção de produtos desenvolvida mais facilmente. Essas informações digitais podem ser distribuídas dentro da empresa, facilitando a tomada de decisões melhores e mais rápidas. $\mathrm{O}$ que também permite uma previsão de tendências aprimorada, já que o design e a amostragem em 3D podem reduzir drasticamente os prazos de entrega e, assim, permitir que as empresas produzam o que os consumidores desejam em tempo hábil.

Vale ressaltar que a transformação digital do fluxo de trabalho de uma marca é um processo iterativo, que envolve tanto a mudança de mentalidade e cultura internas, quanto o conhecimento técnico. Segundo Katrin Ley (2020), os benefícios que acompanham a digitalização do projeto do vestuário na cadeia de valor da moda, incluem:

- Projetos mais precisos e melhor comunicação entre designers e fabricantes, que podem potencialmente levar a produtos projetados de maneira mais eficaz para sua finalidade;

- Menos protótipos, pilotos e amostras, o que significa menos desperdício;

- Prazos significativamente mais curtos, o que pode contribuir para limitar a superprodução e facilitar a fabricação sob demanda.

O relatório setorial The State of Fashion 2020: Coronavirus Update, produzido pela parceria entre o jornal The Business of Fashion e a empresa norte-americana de consultoria McKinsey\& Company, destaca que novos hábitos já estão sendo colocados em prática na indústria da moda. $\mathrm{O}$ estudo aponta um aumento de $84 \%$ do trabalho remoto, $79 \%$ no uso vídeo conferências, e $58 \%$ na flexibilização da jornada de trabalho. Dessa maneira, investir em tecnologia que permita interação eficiente dos funcionários poderá atender às equipes também em longo prazo. O relatório defende que a inovação através da tecnologia foi estendida a toda a cadeia de valor da moda e está aqui para ficar, como o gráfico 01 abaixo demonstra (AMED et al., 2020). 


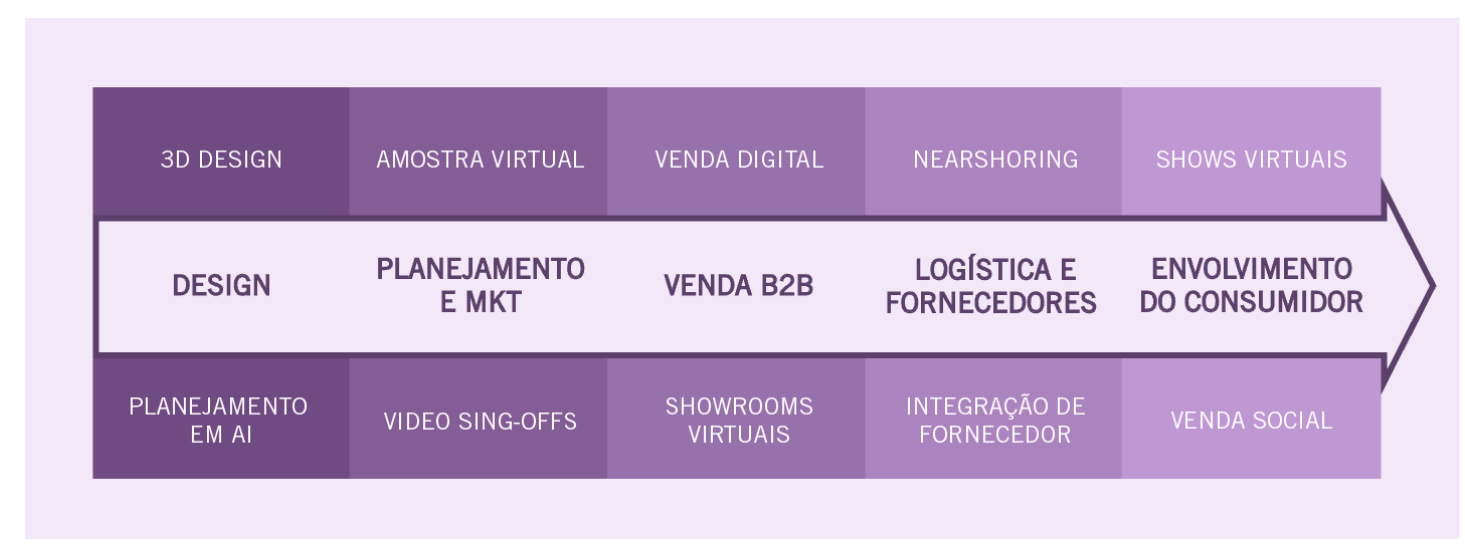

Grafico 01. A ampliação da inovação ao longo da cadeia de valor da moda.

Fonte: Adaptado pelos autores de AMED et al. (2020).

\section{O Impacto no Ensino}

Em uma matéria publicada no site da revista Forbes em maio de 2020, Brooke Roberts-Islam conversa com professores para investigar como a próxima geração de profissionais do setor da moda está sendo preparada para as rápidas transformações tecnológicas que estão acontecendo. É fato que nas mais renomadas faculdades do mundo, incluindo a Parsons School of Design, em Nova York, e o London College of Fashion e Central Saint Martins, ambas em Londres, dispõem de laboratórios que oferecem aos alunos suporte para aprender e criar produtos e experiências, usando softwares e hardwares para design de moda digital e realidade aumentada, porém, em termos curriculares, mantêm uma abordagem para o ensino de design de moda, fundamentada nas principais habilidades manuais do desenho, ilustração e modelagem (ROBERTS-ISLAM, 2020). Ou seja, mesmo que as ferramentas de projeto tenham se tornado digitais na última década, o processo criativo explorado no ensino do design, permanece resolutamente manual. No Brasil, desconhecemos escolas de design de moda que integraram o projeto virtual nessas condições em suas matrizes curriculares.

A indústria tem se esforçando no desenvolvimento digital, e parte das universidades procuram ajudar os estudantes que optam por desenvolver estas habilidades. Contudo, cursos com caráter técnico, como os que são dedicados à formação de técnicos em vestuário, têm absorvido com mais facilidade os sistemas 3D. Isso expõe a visão da divisão entre os profissionais criativos e os técnicos. Entretanto, as soluções de softwares digitais mencionadas requerem conhecimentos de ambos os contextos. Muitos estudantes e profissionais, viram no isolamento e na oferta de acesso gratuito aos softwares e tutoriais durante a crise do COVID-19, uma oportunidade de desenvolvimento pessoal. Porém, não há uma disposição formal para que os educadores sejam treinados nessas soluções de design digital. - Uma grande parte dos educadores com habilidades em Clo3D ou em softwares similares, por exemplo, são autodidatas (ROBERTS-ISLAM, 2020). O 3D tem potencial para ajudar a preparar os negócios de novos criativos. Recentemente, deixar a universidade como designer muitas vezes significa esmagar expectativas em relação ao cotidiano da atividade profissional. Em vez de viver na vanguarda vibrante, dando vida a ideias, o talento jovem está herdando um sério fardo administrativo. No lugar de aproveitar ao máximo sua experiência, as marcas estão pedindo que os designers gastem seu tempo lutando com planilhas e entrada de dados. Isso se traduz em uma perda mensurável de talentos. Com o aumento na agilidade no tratamento de dados que novas tecnologias permitem, é possível "liberar tempo gasto" para o desenvolvimento de trabalhos mais criativos, capazes de alcançar a inovação em diversos níveis.

\section{Considerações Finais}

A pandemia do novo coronavírus impactou fortemente a indústria da moda, forçando as empresas do setor a prescindirem de práticas industriais antigas e criarem estratégias novas para se manterem vivas. Movimentos rápidos para interromper a produção, fechar lojas, encontrar fluxos de receita alternativos 
e identificar novas maneiras de trabalhar se tornaram necessárias para transpor o momento instável das quarentenas. Isso tem levado à aceleração no incremento de novas realidades operacionais que estavam em fase teste até alguns meses atrás. Algumas tecnologias que demoraram a se estabelecer no mercado, como desfiles virtuais, transmissões ao vivo e as mais recentes ferramentas de design 3D, agora estão sendo invocadas para fazer negócios. Esse processo de transformação digital, como apresentado até aqui, inclui soluções voltadas para os processos de criação, produção e consumo. A maior velocidade e o foco na digitalização estão ressignificando processos tradicionais da cadeia produtiva. Os desafios são muitos, e as ações para adaptação parecem ser os próximos obstáculos para a indústria da moda global.

O surgimento de softwares 3D dedicados ao desenvolvimento preciso de peças de vestuário teve um impacto significativo na indústria da moda nos últimos anos. Embora frequentemente usado para visualização e marketing, o potencial dessas ferramentas para transformar a prática de design em si é vasta e deixa clara as vantagens do design 3D para o projeto de roupas são. Toda decisão de projeto tem um impacto na eficiência e na expressão, e esses softwares permitem ao projetista ver em tempo real as consequências das suas decisões. O uso de ferramentas $3 \mathrm{D}$ digitais abre novas oportunidades para a aplicação da prática ao design de moda, articulando novos fluxos e maneiras de trabalhar. Neste artigo, discutimos uma série de ações realizadas pela indústria, pesquisa e ensino, para demonstrar a maneira como as tecnologias digitais estão colaborando para o combate à crise gerada pela pandemia de Covid-19, percebendo como a prática do design se insere neste contexto.

Para lidar com novas restrições, atenuando os danos causados pela pandemia e adaptar-se à piora das condições econômicas, as empresas e a academia devem pensar na inovação em toda a cadeia de valor para proteger o futuro do setor. Acreditamos que é importante que as instituições e organizações ligadas ao campo aproveitem essas inovações para fazer mudanças radicais e duradouras, o que pode conferir diferenciais competitivos no cenário pós-crise. Novas tecnologias e processos bem sucedidos que forem implementados poderão ter um efeito profundo no futuro, fazendo com que todos se familiarizem com os benefícios de novas práticas. Desejamos que essa cadeia produtiva, que é uma das que mais emprega em todo mundo, suporte as adversidades do tempo vigente para viver um momento melhor no futuro.

\section{Virtual Fashion: the digital acceleration process due to the COVID-19 pandemic}

Abstract: The 2020 global pandemic has pushed the fashion system into a new crisis. Although its duration and gravity remain unknown, this industry is only at the beginning of its struggle. In this article, we aim to present and discuss the role of new technological solutions linked to the concept of Industry 4.0 in the face of the crisis generated by the Covid-19 pandemic. The writing of this work was made possible through a systematic review of the literature related to digital technologies with emerging use in the fashion industry and the analysis of recent news related to their possible applications as solutions to the uncertainties of now. The study, carried out between May and August 2020, considers contexts of industry, research, and teaching. It explores a series of examples of technologies in use of contexts, revealing new workflows and opportunities for using 3D software in the Design process. He argues that the advantages of digital technologies to improve the Fashion Design process are particularly evident in the face of this adverse moment that we are experiencing.

Keywords: Fashion Industry; social distance; virtualization; 3D softwares 


\section{Referências}

ABIT. Pesquisa Conjuntural Junho de 2020, 2020. Disponível em:

<https://www.abit.org.br/uploads/arquivos/Pesquisa Conjuntural JUNHO 2020.pdf>

ALDRICH, W. Metric pttern cutting for women's wear. 5. ed. [s.I.] Blackwell, 2008.

AMED, I. et al. The State of Fashion 2020: Coronavirus UpdateThe Business of Fashion and the McKinsey community. [s.l: s.n.]. Disponível em:

$<$ https://www.mckinsey.com/ /media/McKinsey/Industries/Retail/Our Insights/The State of Fashion 2019 A year of awakening/The-State-of-Fashion-2019-final.ashx>.

BLANKS, T. How Sunnei Bucked the Fashion System. Disponível em:

$<$ https://www.businessoffashion.com/articles/tims-take/is-sunnei-the-future-of-milan-fashion>.

Acesso em: 6 ago. 2020.

BOLDT, R. et al. Pattern Design Methods for Non-Conventional Bodies. IOP Conference Series:

Materials Science and Engineering, v. 459, n. 1, p. 012091, 7 dez. 2018.

BOLDT, R. et al. 3D CAD method for smart garments fit prediction. (AUTEX 2019, Ed.)19th

World Textile Conference on Textiles at the Crossroads. Anais...Ghent, Belgium: 2019

BRUNO, F. DA S. A quarta revolução industrial do setor têxtil e de confecção: a visão de futuro para 2030. São Paulo: Esração das Leras e Cores, 2017.

CAMPBELL, J. This Fashion Designer Used Tech To Create A Virtual 3D Fashion Show.

Disponível em: <https://www.gq.com.au/style/news/this-fashion-designer-is-using-tech-to-create-avirtual-3d-fashion-show/news-story/61ce66142f695cfb32af1a0097b0cd80 >. Acesso em: 13 ago. 2020.

CLO VIRTUAL FASHION. USER STORIES: Hear it from actual users who have benefited from

CLO. Disponível em: <https://www.clo3d.com/users/story>. Acesso em: 3 jun. 2018.

CLO VIRTUAL FASHION. CLO x Balmain x Shudu Collaboration Revealed. Disponível em: <https://www.clo3d.com/company/newsdetail/101>. Acesso em: 13 ago. 2020.

COSTA, T. N.; PIERRE, R.; LUISA, T. Uso Da Digitalização 3D E Da Parametrização De Medidas Antropométricas Para Produção De Moldes Personalizados Para O Vestuário 3D Scanning and Parameterization of Anthropometric Measures in Custom Molds Production for Clothing. Educação Gráfica, v. 19, n. 2, 2015.

DOUPNIK, E. Ordre Showroom Deploys VR for Buyers Unable to Attend Fashion Shows WWD. Disponível em: <https://wwd.com/business-news/technology/ordre-showroom-virtual-reality1202627011/>. Acesso em: 14 ago. 2020.

DRATH, R.; HORCH, A. Industrie 4.0: Hit or hype? [Industry Forum]. IEEE Industrial Electronics Magazine, v. 8, n. 2, p. 56-58, 2014.

ELGAMMAL, A. et al. CAN: Creative Adversarial Networks, Generating "Art" by Learning About Styles and Deviating from Style Norms. International Conference on Computational Creativity, $n$. IccC, p. 1-22, 21 jun. 2017.

FFW. Moda.com: elegemos as melhores apresentações da temporada digital. Disponível em: $<$ https://ffw.uol.com.br/noticias/moda/moda-com-elegemos-as-melhores-apresentacoes-datemporada-digital/>. Acesso em: 22 jul. 2020.

FRIEDMAN, V. Is This the Future of the Fashion Show? Disponivel em:

<https://www.nytimes.com/2020/05/02/fashion/coronavirus-digital-fashion-show.html>. Acesso em: 22 jun. 2020.

HERMANN, M.; PENTEK, T.; OTTO, B. Design principles for industrie 4.0 scenarios. Proceedings of the Annual Hawaii International Conference on System Sciences, v. 2016- March, p. 39283937, 2016.

HONG, Y. A. N. et al. Application of 3D-TO-2D garment design for atypical morphology : a design case for physically disabled people with scoliosis. v. 69, n. 1, p. 59-64, 2018. 
KANG, T.-S.; LEE, D.-Y.; KIM, J. Production of Digital Fashion Contents based on Augmented Reality Using CLO 3D and Vuforia. Journal of the Korea Computer Graphics Society, v. 26, n. 3, p. 21$29,2020$.

KHERDE1, A.; JAWADE2, P. Novel Approach to Fashion Design using Artificial Intelligence. International Journal of Science and Research, v. 8, 2018.

KHOSLA, V. Prada picks Bengaluru-based BigThinx for its fashion tech accelerator programme - The Economic Times. Disponível em: <https://economictimes.indiatimes.com/smallbiz/startups/newsbuzz/prada-picks-bengaluru-based-bigthinx-for-its-fashion-tech-acceleratorprogramme/articleshow/73901212.cms>. Acesso em: 14 ago. 2020.

KIM, J. et al. Prediction and categorization of fabric drapability for 3D garment virtualization. International Journal of Clothing Science and Technology, v. 32, n. 4, p. 523-535, 2020.

LECTRA. Cordeiro Campos: consumer stories. Disponível em:

<https://www.lectra.com/en/customer-stories/cordeiro-campos>. Acesso em: 8 ago. 2018.

LEY, K. No TitlePromising areas for innovation in COVID-19 times: Fashion for Good points to solutions in key focus areas to strengthen the fashion industry. Disponível em: $<$ https://medium.com/fashion-for-good/promising-areas-for-innovation-in-covid-19-times-part-3digital-acceleration-3fe15068ac54>. Acesso em: 6 ago. 2020.

LIU, K. et al. Fit evaluation of virtual garment try-on by learning from digital pressure data. Knowledge-Based Systems, v. 133, p. 174-182, 2017.

LIU, K. et al. Garment Fit Evaluation Using Machine Learning Technology. In: THOMASSEY, S.; ZENG, $X$. (Eds.). . Artificial Intelligence for Fashion Industry in the Big Data Era. [s.l.] Springer Series in Fashion Business, 2018. p. 273-288.

LOUIS VUITTON. Lightning: uma heroína virtual. Disponível em: <https://br.louisvuitton.com/porbr/articles/lightning-uma-heroina-virtual>. Acesso em: 7 maio. 2019.

LU, S.; MOK, P. Y.; JIN, X. A new design concept: 3D to 2D textile pattern design for garments. CAD Computer Aided Design, v. 89, p. 35-49, 2017.

MA, Y. et al. Knowledge enhanced neural fashion trend forecasting. ICMR 2020 - Proceedings of the 2020 International Conference on Multimedia Retrieval, p. 82-90, 2020.

$\mathrm{MAO}$, A. et al. A multi-disciplinary strategy for computer-aided clothing thermal engineering design. CAD Computer Aided Design, v. 43, n. 12, p. 1854-1869, 2011.

MCQUILLAN, H. Digital 3D design as a tool for augmenting zero-waste fashion design practice.

International Journal of Fashion Design, Technology and Education, v. 13, n. 1, p. 89-100, 2020.

MENG, Y.; MOK, P. Y.; JIN, X. Computer aided clothing pattern design with 3D editing and pattern alteration. Computer-Aided Design, v. 44, n. 8, p. 721-734, ago. 2012.

OPTITEX. Optitex. Disponível em: <optitex.com/lp/o18-i>. Acesso em: 3 jun. 2018.

PAGE, A. Creative pattern technology. International Journal of Fashion Design, Technology and Education, v. 6, n. 2, p. 89-98, jul. 2013.

ROBERTS-ISLAM, B. Fashion Education And Startup Accelerators: How COVID-19 Is Changing The Fashion Industry. Disponível em:

<https://www.forbes.com/sites/brookerobertsislam/2020/04/30/fashion-education-and-startupaccelerators-how-covid-19-is-changing-the-fashion-industry/\#4e7e119c49ed>. Acesso em: 2 maio. 2020.

SADAT, A.; SAYEM, M. Advances in Virtual Prototyping: Opportunities for Clothing Manufacturers. n. December, 2015.

SCHWAB, K. A quarta revolução industrial. 1. ed. São Paulo: Edipro, 2016.

SEGATTI, A. L. Ralph \& Russo apresenta sua primeira coleção digital para a Alta Costura

Outono 2020. Disponível em: <https://www.revistalofficiel.com.br/fashion-week/ralph-russo-haute- 
couture>. Acesso em: 6 ago. 2020.

SILVEIRA, I.; SILVA, G. Conhecimentos Dos Modelistas Catarinenses E Os Softwares Utilizados Nos Setores De Modelagem Do Vestuário. Modapalavra E-periódico, v. 4, n. 7, p. 12-26, 2011.

SOCHA, M. Tommy Hilfiger Has Avatars, Holograms at the Ready. WWD, 2020.

VIEIRA, L. Hanifa agita o mercado da moda ao lançar a coleção com desfile 3D. Disponível em: <https://revistaglamour.globo.com/Moda/Fashion-news/noticia/2020/05/hanifa-agita-o-mercadoda-moda-ao-lancar-colecao-com-desfile-3d.html>. Acesso em: 3 ago. 2020.

WONG, W. K.; CHAN, C. K. An Artificial Intelligence Method for Planning the Clothing Manufacturing Process. Journal of the Textile Institute, v. 92, n. 2, p. 168-178, jan. 2001. 